\title{
A method for estimating time-frequency characteristics of compact binary mergers to improve searches for inspiral, merger and ring-down phases separately
}

\author{
Chad Hanna $^{1,2}$, Miguel Megevand ${ }^{2}$, Evan Ochsner ${ }^{3}$ \\ and Carlos Palenzuela ${ }^{2,4}$ \\ ${ }^{1}$ LIGO Laboratory, California Institute of Technology MS 18-34 Pasadena, CA 91125, USA \\ ${ }^{2}$ Department of Physics and Astronomy, Louisiana State University, Baton Rouge, LA 70803, \\ USA \\ ${ }^{3}$ Department of Physics, University of Maryland, College Park, MD 20742, USA \\ ${ }^{4}$ Max-Planck-Institut fur Gravitationsphysik, Albert-Einstein-Institut, Golm, Germany \\ E-mail: channa@phys.1su.edu,megevand@phys.1su.edu, evano@umd.edu and carlos@1su.edu
}

Received 26 January 2008, in final form 27 October 2008

Published 16 December 2008

Online at stacks.iop.org/CQG/26/015009

\begin{abstract}
Recent advances in the description of compact binary systems have produced gravitational waveforms that include inspiral, merger and ring-down phases. Comparing results from numerical simulations with those of post-Newtonian, and related, expansions has provided motivation for employing post-Newtonian waveforms in near merger epochs when searching for gravitational waves and has encouraged the development of analytic fits to full numerical waveforms. Until searches employ full waveforms as templates, data analysts can still conduct separate inspiral, merger and ring-down searches. Improved knowledge about the end of the inspiral phase, the beginning of the merger and the ring-down frequencies will increase the efficiency of searches over each phase separately without needing the exact waveform. We will show that knowledge of the final spin, of which there are many theoretical models and analytic fits to simulations, may give an insight into the time-frequency properites of the merger. We also present implications on the ability to probe the tidal disruption of neutron stars through gravitational waves.
\end{abstract}

PACS numbers: $04.80 . \mathrm{Nn}$, 97.60.Lf

\section{Introduction}

Gravitational radiation emitted during the inspiral, merger and ring-down of two compact objects with a total mass below $\sim 100 M_{\odot}$ is a likely detection source for interferometric 
gravitational-wave detectors [1]. Maximal signal-to-noise ratio (SNR) is achieved in searches for compact binary coalescence with matched filtering banks of template waveforms [2-6] allowing faint signals to be detected reliably. The SNR depends on having a template waveform that matches the true signal with high fidelity [2]. It is important when in the binary evolution the template matches well. If analysts doubt that a given waveform captures a particular phase of the evolution they may choose to omit that portion in matched filter searches. For example, a search may only choose to look for the inspiral phase of binary evolution and leave the merger and ring-down phases for different searches. Should the analyst doubt the model of a particular phase it may be prudent to abandon matched filtering altogether in favor of a more generic algorithm for unmodeled sources. However even generic methods will still benefit from a best guess at the signals time-frequency properties.

Numerical simulations improve our knowledge of the full waveforms emitted during the inspiral, merger and ring-down (IMR) phases of binary black hole coalescence [7-9], yet they still do not cover an adequate parameter space for constructing filter banks [9, 10]. Many have considered hybrid waveforms that patch together PN and numerical simulations $[7-9,11,12]$ as data analysis templates. Others have introduced a phenomenological fourthorder correction to make effective one-body (EOB) waveforms match numerical relativity results near the merger [7, 11] known as pseudo 4PN (p4PN).

There is no doubt that full IMR templates will eventually be used for detection searches. But, before data analysts implement full inspiral-merger-ring-down detection templates, or in conjunction with such an analysis, analysts may still handle the three binary coalescence epochs-inspiral, merger and ring-down-as separate searches. We will show that extending a recent calculation [13], which has power to predict the final spin of a binary merger, can provide insight into the time-frequency characteristics of inspiral, merger and ring-down in a self-consistent way that can facilitate data analysts in this endeavor. With caution analysts may apply the results here to yet unsimulated systems.

The calculation of the final spin given in [13] contains an implicit reference to the innermost-stable-circular orbit (ISCO) of a test particle orbiting the Kerr black hole that results from the merger. This ISCO-hereafter the BKL ISCO-defines a merger epoch that lasts until the Kerr light ring computed in the same way. The time-of-flight of the test particle to free-fall on its geodesic between the ISCO and light-ring seems to predict the duration of the merger in the corresponding binary simulations. Thus with the BKL model we have natural time-frequency boundaries for separate inspiral, merger and ring-down searches.

We note that the work of [13] in spirit is similar to the earlier work on the effective one-body approach to general relativistic two-body dynamics [15]. The key difference is the mapping of the final binary spacetime onto a Kerr rather than Schwarzschild spacetime for initially non-spinning binaries in the case of [13]. We feel that a similar analysis as presented in this work may be done within the EOB framework, yet we prefer to stick with the simple model in [13].

We will first recap the formalism presented in [13] and then discuss how calculations may be extended to provide a natural cut-off for the inspiral phase, the time-frequency volume for the merger epoch and the ring-down frequencies. The final section will describe how probing the neutron star tidal disruption could be modified by interpreting the BKL ISCO as a true innermost-stable-circular orbit.

\section{Formalism}

The classification of inspiral, merger and ring-down phases for near equal mass configurations is arbitrary. There is not a unique way to define when the inspiral phase ends and the 
merger begins for much of the parameter space. However, separate IMR phase searches must artificially separate the phases by some criteria. Theoretical guidance exists for extreme mass ratio binaries, where the system can be viewed as a test particle orbitting a larger object with well-defined spacetime, such as Schwarzschild or Kerr spacetime. In the test particle limit the end of the inspiral phase is generally taken to be the innermost-stable-circular orbit (ISCO). For comparable mass systems defining the ISCO may be fruitless but it can nevertheless be defined or dismissed by waveform models and numerical relativity [7, 17, 18], albeit with non-trivial differences in the numbers obtained from different methods. Some LIGO searches for non-spinning systems have taken the conservative approach to using the ISCO defined for a test particle orbiting a Schwarzschild black hole whose mass is the total mass of the binary system to mark the end of inspiral for all non-spinning binary systems regardless of mass ratio. This is conservative because it seems to under-predict the ISCO estimates for equal mass binaries.

Choosing a frequency that may or may not represent an ISCO for inspiral searches is not the only consideration. The templates used must be faithful throughout the frequency range chosen. In this section, we show that the calculations of [13] can be extended to provide a merger transition frequency for inspiral searches, where PN templates are valid, and additionally they provide an estimate for the time-frequency nature of the merger. This combined with an effective ring-down frequency obtained from the final spin of the system gives a simple and unified way of calculating the properties of the IMR phases without having the details of the full waveform. With caution this could be applied to regions of parameter space not yet simulated numerically and can be used in lieu of a search for the full waveform to optimally stitch together already existing inspiral, merger and ring-down searches.

We will begin by recapping the formalism presented in [13] to obtain the final spin of a binary system. This calculation involves computing the ISCO of a test particle orbiting the final black hole. We will argue that this frequency could be used to define the onset of merger; beyond which we will show that the duration of the merger for a given binary system seems to be well predicted by the time-of-flight of a test particle orbiting the final Kerr black hole. It goes almost without mention that the ring-down frequency can be found from the final spin and the $l=m=2, n=0$ mode is actually quite close to the frequency of the last stable photon orbit of the final Kerr black hole.

\subsection{Defining the merger epoch}

The ISCO solution for a test particle orbiting a Kerr black hole is [20]

$$
\begin{aligned}
& Z_{1} \equiv 1+\left(1-\frac{a_{f}^{2}}{M^{2}}\right)^{1 / 3}\left[\left(1+\frac{a_{f}}{M}\right)^{1 / 3}+\left(1-\frac{a_{f}}{M}\right)^{1 / 3}\right] \\
& Z_{2} \equiv\left(3 \frac{a_{f}^{2}}{M^{2}}+Z_{1}^{2}\right)^{1 / 2} \\
& r_{\text {ISCO }}=M\left\{3+Z_{2} \mp\left[\left(3-Z_{1}\right)\left(3+Z_{1}+2 Z_{2}\right)\right]^{1 / 2}\right\},
\end{aligned}
$$

where $M$ is the total mass of the black hole and $a_{f}$ is the angular momentum. In [13], assuming the amount of mass and angular momentum radiated beyond the ISCO is small, the following implicit formula for the final angular momentum of a black hole $a_{f}$ with component spins aligned with the orbit is calculated,

$$
\frac{a_{f}}{M}=\frac{L_{\mathrm{orb}}}{M^{2}}\left(q, \frac{r}{M}=\frac{r_{\mathrm{ISCO}}}{M}, \frac{a_{f}}{M}\right)+\frac{q^{2} \chi_{1}+\chi_{2}}{(1+q)^{2}},
$$




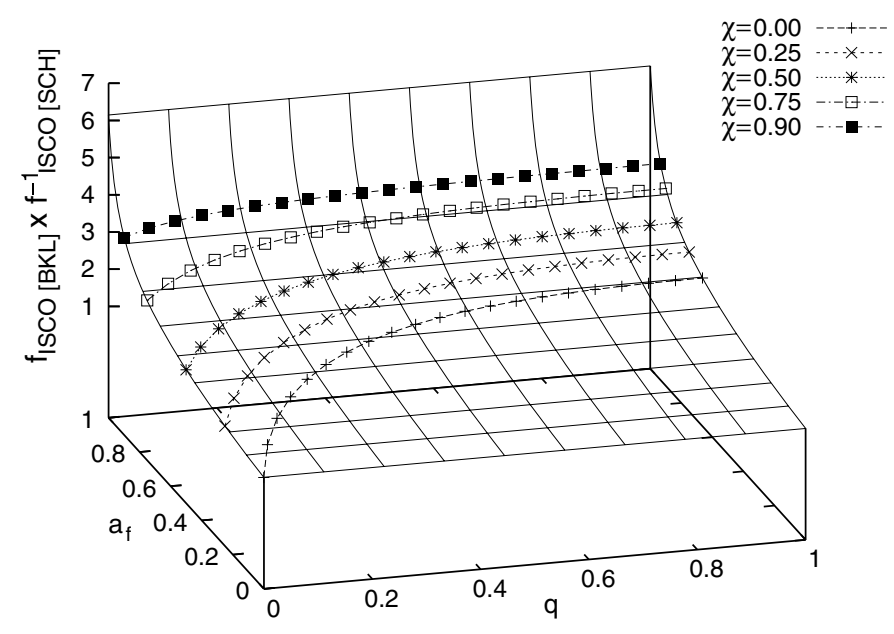

Figure 1. The surface of solutions of the frequencies at the ISCO as a function of the mass ratio $q$ and the final spin $a_{f}$ for components with spins that are aligned with the orbital angular momentum. Also shown are curves corresponding to the solution of the equal spin case $\chi_{1}=\chi_{2}=\chi$.

where $\chi_{i}=a_{i} / m_{i}, q=m_{1} / m_{2} \in[0,1]$ and $M=m_{1}+m_{2}$ is the total mass. The implicitly found $a_{f}$ agrees well with numerical simulations [13] and the analysis can be modified to include arbitrary spin angles. $L_{\text {orb }}$ is the orbital angular momentum contribution calculated from the orbital angular momentum of a particle at the ISCO of a Kerr black hole with spin parameter $a_{f}$, which has the following expression [20],

$$
\frac{L_{\mathrm{orb}}}{M^{2}}\left(q, \frac{r}{M}, \frac{a_{f}}{M}\right)=\frac{q}{(1+q)^{2}} \frac{ \pm\left(r^{2} \mp 2 a_{f} M^{1 / 2} r^{1 / 2}+a_{f}^{2}\right)}{M^{1 / 2} r^{3 / 4}\left(r^{3 / 2}-3 M r^{1 / 2} \pm 2 a_{f} M^{1 / 2}\right)^{1 / 2}},
$$

where the upper/lower signs correspond to prograde/retrograde orbits. In order to agree with numerical simulations this function has to be evaluated at $r=r_{\text {ISCO }}$ given by equation (1).

In order to get the gravitational wave frequency at the ISCO we use the coordinate angular velocity of a circular orbit [20],

$$
\Omega= \pm \frac{M^{1 / 2}}{r^{3 / 2} \pm a_{f} M^{1 / 2}}
$$

with $a_{f}$ given by the implicit equation (2). The gravitational-wave frequency at a given radius is then $f=\Omega / \pi$, and so we define $f_{\mathrm{ISCO}[\mathrm{BKL}]}$ as the frequency obtained by solving the system of equations (1-4) at the Kerr ISCO radius.

The solution space for $f_{\mathrm{ISCO}[\mathrm{BKL}]}$ can be written as a function of the final unknown spin $a_{f}$. For convenience, we prefer to extend it as a surface parametrized by $\left(a_{f}, q, \chi\right)$, where $\chi_{1}=\chi_{2} \equiv \chi$, as is shown in figure 1. This way the mass ratio dependence of the lines corresponding to different individual spins can be seen explicitly. For the equal mass case without spin the approximate expression for the Kerr test particle ISCO frequency is

$$
f_{\mathrm{ISCO}[\mathrm{BKL}]}(M, q) \approx\left(0.8 q^{3}-2.6 q^{2}+2.8 q+1\right) \times \frac{1}{\pi 6^{3 / 2} M},
$$

which can be compared to the Schwarzschild test particle ISCO $f_{\mathrm{ISCO}[\mathrm{SCH}]}=\pi^{-1} 6^{-3 / 2} M^{-1}$.

Once the final spin is known it is possible to compute the last circular photon orbit ("light ring') and the quasi-normal mode ring-down frequencies [16]. The light ring for a Kerr black hole is [20] 


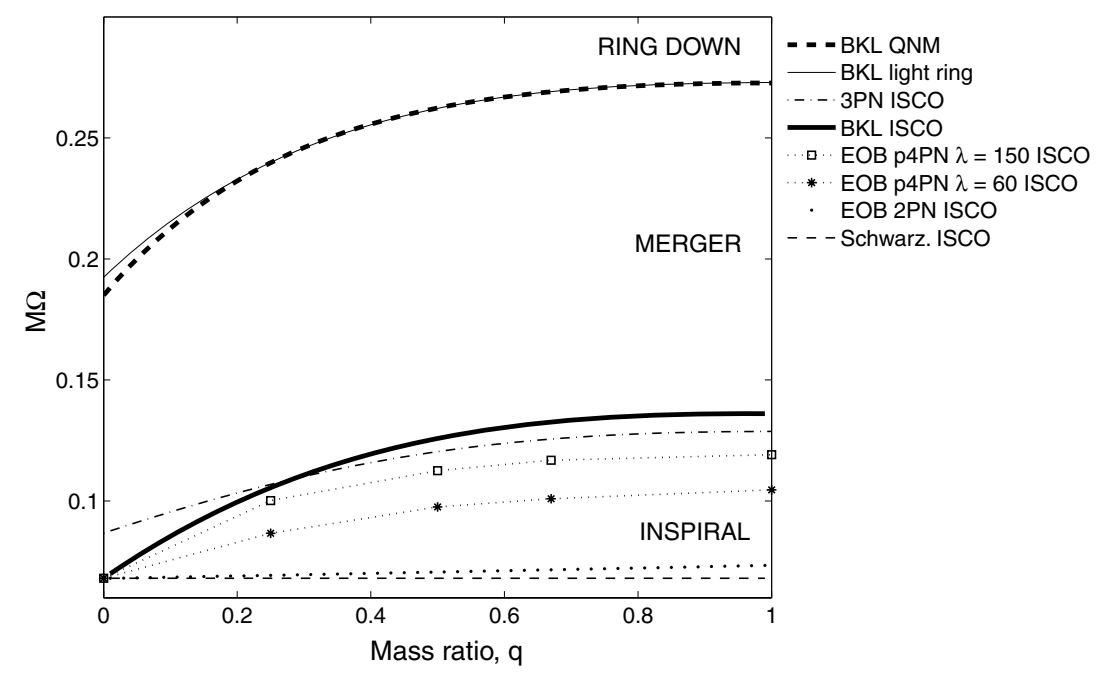

Figure 2. Defining the inspiral, merger and ring-down epochs as a function of mass ratio for nonspinning binaries. Also plotted are ISCO estimates from other methods such as the PN minimum energy conditions and EOB models. The next section will show that one can also estimate the duration of the merger, meaning that a good estimate exists for the time-frequency volume of a merger without needing the details of the waveform.

$$
\begin{aligned}
& r_{\text {light }}=2 M\left\{1+\cos \left[2 / 3 \cos ^{-1}\left(\mp a_{f} / M\right)\right]\right\} \\
& \Omega_{\text {light }}= \pm \frac{M^{1 / 2}}{r_{\text {light }}^{3 / 2} \pm a_{f} M^{1 / 2}}
\end{aligned}
$$

The light ring frequencies can be compared with an approximate fit to the quasinormal mode ring-down frequencies as a function of the final spin [25-27]. For the $l=2, m=2, n=0$ mode we have

$$
\Omega_{\mathrm{QNM}} \approx \frac{0.5}{M}\left(1-0.63\left(1-a_{f}\right)^{0.3}\right) .
$$

Other ring-down modes could be computed as necessary.

Figure 2 shows the inspiral, merger and ring-down epochs defined by the BKL calculations. For comparison we have plotted ISCOs computed from the minimum energy conditions at the 3PN order [19] as well as $2 \mathrm{PN}$ and p4PN EOB ISCOs $[15,16]$ and an analytic fit to the ring-down frequencies from [25-27].

In the following sections we will argue that: (1) the choice of the BKL ISCO is sensible for the cut-off frequency of the inspiral phase and (2) the time-frequency characteristics of the merger are set by calculating the time-of-flight for a test particle infalling from the ISCO of the final Kerr black hole to the last stable photon orbit along its geodesic.

\subsection{Is the BKL ISCO a sensible choice for the end of the inspiral phase?}

As mentioned previously, there is ambiguity on how to define when the inspiral phase ends. However, what is really necessary is the characteristic frequency at which a given template waveform ceases to resemble the physical systems. Some searches for low mass binary systems of non-spinning component masses use the ISCO of a test particle orbiting 
a Schwarzschild black hole [14]. Other searches use the ISCOs calculated from explicit PN energy considerations, and some abandon the use of an ISCO altogether and use the Schwarzschild light ring as a termination frequency [15].

Recall that for low mass ratio $(q \sim 0)$ systems of non-spinning objects, the Schwarzschild test particle limit is a good approximation for the expected ISCO frequency since the merger product will be a Schwarzschild-like black hole. However, for systems with comparable masses and/or spin the true ISCO frequency may be different since the non-trivial contribution from the orbital angular momentum will have a strong impact on the final black hole's spin, and the spacetime in the near merger epoch. Many have addressed how well various PN approximants stay faithful to numerical relativity solutions [7, 11, 41-45]. Most approximants stay faithful through the Schwarzschild ISCO frequency [43]. Some approximants fare extremely well beyond this point [43].

The fact that some approximants do remain faithful far beyond the Schwarzschild ISCO leads us to examine using the ISCO frequency described in the previous section, equation (5), as the termination frequency for inspiral data analysis. As shown, this frequency is consistent with some of the PN and EOB models for predicting the ISCO in the equal mass regime (figure 2) and is consistent with exact solutions in the test particle limit. It also has the advantage of being waveform model, or fit, independent (based on first principles) and is easy to calculate. We will conservatively model the impact of possible phase errors incurred by using an approximant that does not quite match numerical relativity results in our assessment.

We use the stationary phase approximation waveforms, which are often employed in searches, and can be defined as

$$
\tilde{h}(f) \propto f^{-7 / 6} \mathrm{e}^{\mathrm{i} \Psi(f ; M, \mu)},
$$

where $M$ is the total mass and $\mu$ is the reduced mass [14]. Such templates ignore amplitude corrections beyond first order. In fact, simulations show that the amplitude may differ significantly from PN waveforms near merger, but we ignore those complicating effects here. In order to ascertain the phase fidelity we turn to comparisons given in [11] which show for equal mass binary simulations the phase difference between numerical results and 3.5 PN waveforms as a function of frequency. The figures in [11] indicate a good fidelity in phase through the Schwarzschild ISCO frequency. However substantial phase errors may accumulate between the Schwarzschild and BKL ISCO frequencies. In order to accurately predict the impact of integrating PN waveforms to the BKL ISCO we decided to model the possibility that the secular phase errors could be $\pm \pi$ or more radians between the Schwarzschild and BKL ISCOs. We propose calculating the SNR ratio of an ensemble of waveforms where phase errors are allowed to accumulate linearly between the Schwarzschild ISCO $f_{2}(M, q)$ and the BKL ISCO $f_{1}(M, q)$ given by,

$\frac{\operatorname{SNR}_{f_{1}}}{\operatorname{SNR}_{f_{2}}}=\left\langle\left(\int_{f_{\text {low }}}^{f_{1}} \frac{\mathrm{e}^{\pi \mathrm{i} R_{n}\left(f_{1}-f_{2}\right) / f_{1} \Theta\left(f_{1}-f_{2}\right)} \mathrm{d} f}{f^{7 / 3} S_{n}(f)}\right)^{1 / 2}\left(\int_{f_{\text {low }}}^{f_{2}} \frac{\mathrm{d} f}{f^{7 / 3} S_{n}(f)}\right)^{-1 / 2}\right\rangle$,

where $R_{n}$ is a normally distributed variable with mean 0 and variance $1, f_{\text {low }}$ is $40 \mathrm{~Hz}[6]^{5}$.

We note that the above calculation is a conservative estimate of the SNR gain for two reasons: (1) template with different parameters may match the numerical waveforms better, producing better phase agreement. Since search results are maximized over SNR this is

5 We take $S_{n}(f)$ to be approximated analytically as [34]

$$
S_{n}(f) \approx 9 \times 10^{-46}\left[\left(\frac{4.49 f}{150}\right)^{-56}+0.16\left(\frac{f}{150}\right)^{-4.52}+0.32\left(\frac{f}{150}\right)^{2}+0.52\right] .
$$




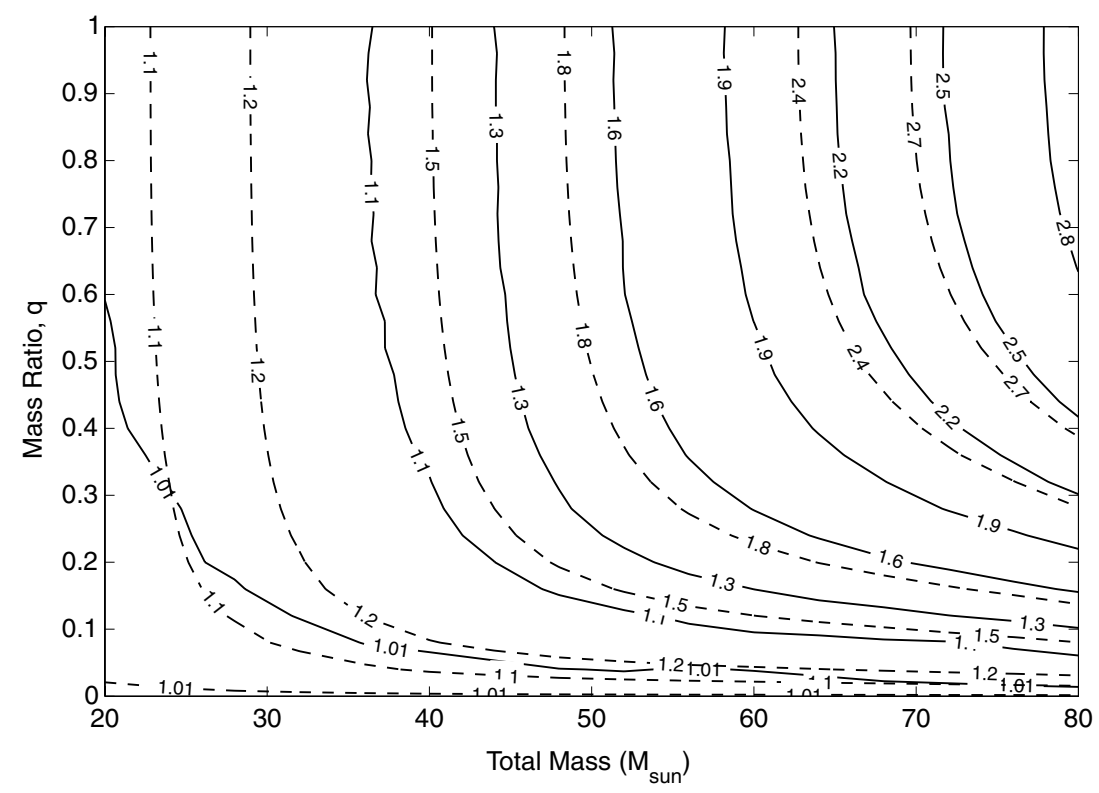

Figure 3. The SNR ratio defined by equation (10) for the non-spinning case $\chi=0$. The solid lines include phase errors of the form (10) whereas the dashed lines assume perfect phase coherence for comparison.

an important possibility. (2) The phase errors do not accumulate linearly between these frequencies, most of the phase errors occur near the BKL ISCO. Using the $\{M, q\}$ dependent ISCO frequencies as $f_{1}(M, q)$ and the Schwarzschild ISCO frequencies as $f_{2}(M)$ we plot the expected SNR ratio for initial LIGO [35] in figure 3, which shows that for some total mass and mass ratio combinations there is an appreciable gain in SNR by integrating to the new ISCO frequencies despite modeled phase errors. This calculation is not intended to suggest that SPA templates cut at the BKL ISCO produce the optimal gain in SNR, since any higher frequency cut-off would likely improve the SNR. Instead, it aims to simply illustrate the effect of integrating to the BKL ISCO as a function of $M$ and $q$ for present searches. The mass range is limited to $80 M_{\odot}$ so that at least two cycles exist in the waveform between $40 \mathrm{~Hz}$ and the Schwarzschild ISCO frequency.

\subsection{Estimating the time-frequency volume of the merger}

It has been shown $[29,30,32]$ to be useful to understand unmodeled time-frequency evolutions in gravitational wave analysis in terms of time-frequency volumes, $\Delta f \Delta t$. This concept can provide insight into the merger epochs of various systems. We will consider three timescales for the merger: (1) the Newtonian free-fall time scale $t_{\mathrm{ff}}$, (2) the quadrupole radiation time scale $t_{\text {quad }}$ and (3) the time scale of a test particle following its geodesic just inside the ISCO of the Kerr spacetime (corresponding to the merger product). For comparison we also plot the timefrequency volumes found from the figures in [16]. The Newtonian free-fall time-frequency volume is

$$
\Delta f_{\mathrm{ISCO}} \Delta t_{\mathrm{ff}}=\frac{2^{-3 / 2}}{\sqrt{M \Omega_{\mathrm{ISCO}}}} .
$$




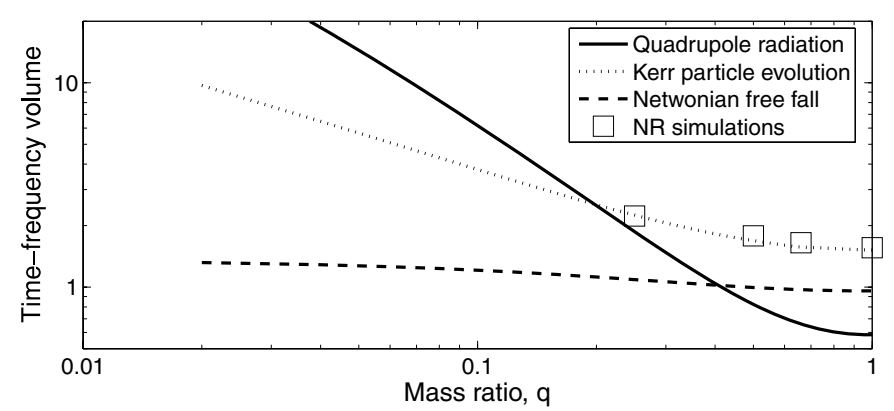

Figure 4. An estimate of the time-frequency volume for the merger epoch of non-spinning binary black holes. Numerical relativity results agree well with the time-frequency volume of a test particle falling into the merged Kerr black hole from a circular orbit just inside the ISCO.

The quadrupole time-frequency volume is calculated by assuming that an ISCO does not exist and that the system continues to be driven by radiation. To calculate this we use the quadrupole approximation to the inspiral waveform given by [31], which gives the frequency evolution as,

$$
M \Omega=\left[\frac{5(1+q)^{2}}{256 q} \frac{M}{t-t_{o}}\right]^{3 / 8}
$$

This leads to the time-frequency volume,

$$
\begin{aligned}
\Delta f_{\mathrm{BKL} \text { ISCO }} \Delta t_{\text {quad }}= & \frac{5(1+q)^{2}}{256 \pi q}\left[\left(M \Omega_{\mathrm{ISCO}}\right)^{-8 / 3}-\left(M \Omega_{\text {light }}\right)^{-8 / 3}\right] \\
& \times\left[\left(M \Omega_{\text {light }}\right)-\left(M \Omega_{\mathrm{ISCO}}\right)\right],
\end{aligned}
$$

where the ISCO frequencies and light ring frequencies are obtained from the BKL approximation as in figure 2 .

The merger evolution will not be purely dominated by radiation by virtue of the fact that there are no circular orbits below the ISCO. The numbers obtained by this estimate should greatly over-predict the time scale for extreme mass ratios where particles would take an asymptotically infinite time to fall in. The free-fall time scale will under-predict the time since the system may still complete some (unstable) orbits before merging. The estimate that agrees best with numerical relativity simulations is the infall time of a test particle falling from a circular orbit just below the ISCO to the light ring of the the merged Kerr black hole. Figure 4 shows the time-frequency volume for the merger epochs as a function of mass ratio for non-spinning binaries. The figure shows that the numbers taken from the simulations presented in [16] agree well with the predictions from the test particle in the Kerr spacetime. By these estimates the mergers from yet unsimulated evolutions (low mass ratio) are likely to have the largest time-frequency content and searches may benefit from the numerical simulations of these [30]. This information should be useful in conducting IMR searches and also in guiding the construction of analytic full waveform models. It, is of course, possible to repeat this analysis for arbitrary spin configurations using the above prescription and others $[13,21,22,33]$. We will leave further discussion of these matters to future work and will spend the rest of this work considering the implications of using the ISCO found from the above set of equations as a termination frequency for PN waveforms in initial LIGO data analysis. 


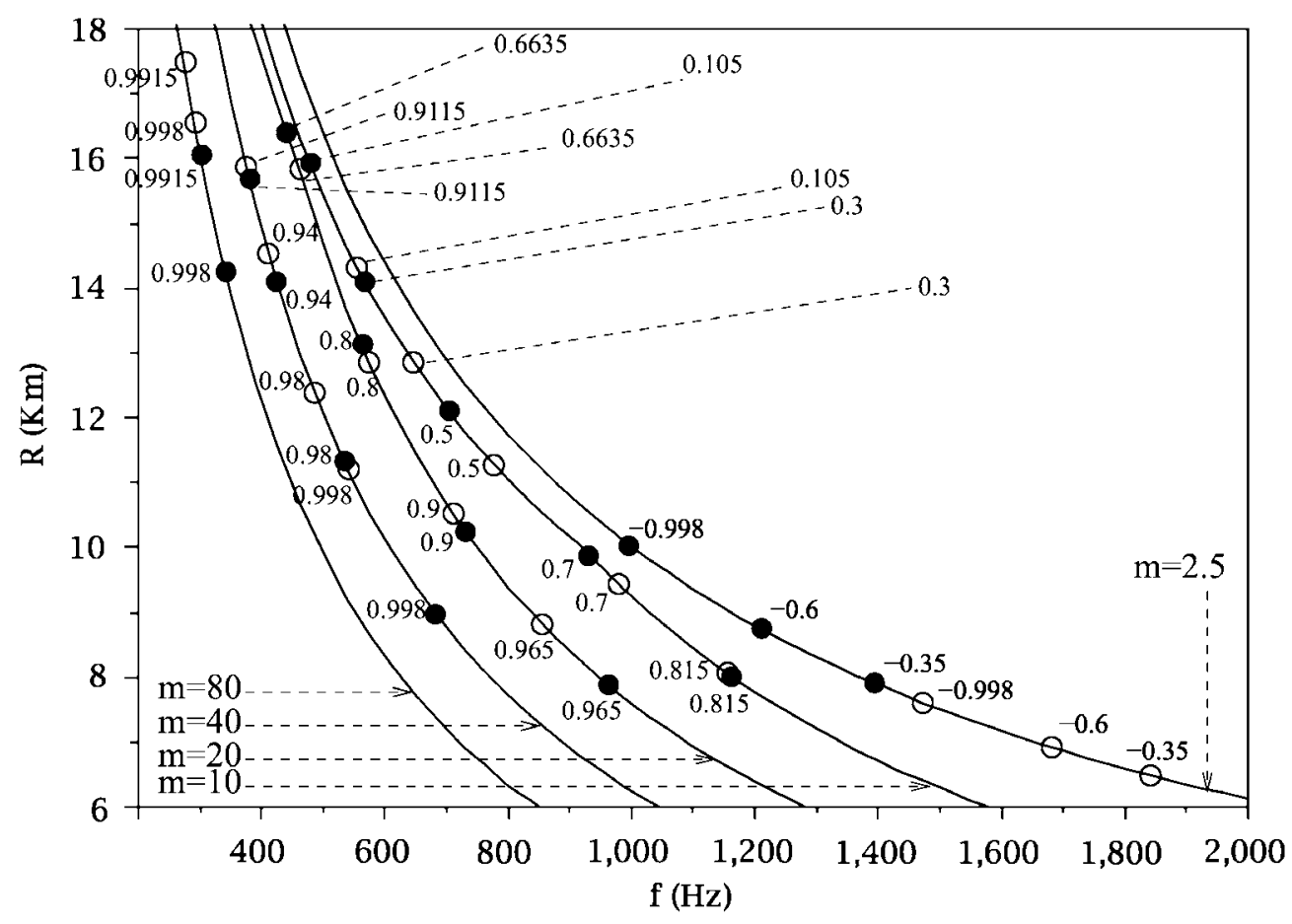

Figure 5. Radius of the neutron star versus the disruption frequency. The circles indicate the points at which the plunge would occur; the filled circles correspond to $f_{\text {plunge }}$ as calculated in [36], included here for comparing. The value of $\chi_{\mathrm{BH}}$ is indicated next to the corresponding circle where possible, otherwise a dashed line was used to connect the value to the circle. Each curve is labeled with the value of the $\mathrm{BH}$ mass: $m_{\mathrm{BH}}=2.5-80 M_{\odot}$.

\section{Probing the tidal disruption of neutron stars}

If the BKL ISCO truly marks the transition from an inspiral, radiation-dominated evolution to a dynamical one then it does have some consquence for the ability to probe the tidal disruption of neutron stars through gravitational waves. Estimation of gravitational-wave frequencies for the tidal disruption of NS-BH binaries may be useful for determining various properties of the neutron star, such as the radius $[36,37]$. Knowing the radius of the neutron star would, in turn, provide information about its equation of state [38, 39]. However, as pointed out in [36], it would be difficult to extract information about the disruption unless it occurs before the binary reaches the ISCO. If the disruption occurs afterward, its signature in the produced gravitational waves might be too weak for extracting accurate information. Thus, the 'useful' cases are those for which the tidal-disruption frequency is less than the frequency at the plunge $\left(f_{\text {td }}<f_{\text {plunge }} \equiv f_{\text {ISCO }}\right)$.

A more accurate calculation of $f_{\text {plunge }}$ would imply a better estimate of the range of NS-BH binaries for which the disruption is more plausible to be measured. In this section we show how that range changes when the formalism of section 2 is applied, as compared to that in [36].

The frequencies obtained in [36] correspond to the extreme mass ratio case, $q=0$, and non-spinning neutron star, $\chi_{\mathrm{NS}}=0$. In figure 5 we reproduce those results (compare to figure 2 of [36]), together with the values obtained using the formalism presented in this work. 
Table 1. BKL frequencies for the same parameters as models A-F of [40]. These values are consistent with the NR results - that is the BKL ISCO frequency is higher than the observable $f_{\text {cut }}$ from the simulations [40].

\begin{tabular}{lllll}
\hline Model & $q$ & $M\left(M_{\odot}\right)$ & $f_{\mathrm{BKL}}(\mathrm{kHz})$ & $f_{\text {cut }}$ from [40] \\
\hline A & 0.327 & 5.277 & 1.38 & 1.16 \\
$\mathrm{~B}$ & 0.327 & 5.244 & 1.39 & 1.41 \\
$\mathrm{C}$ & 0.328 & 5.311 & 1.37 & 0.92 \\
$\mathrm{D}$ & 0.392 & 4.623 & 1.65 & 1.14 \\
$\mathrm{E}$ & 0.392 & 4.594 & 1.66 & 1.40 \\
$\mathrm{~F}$ & 0.281 & 5.929 & 1.18 & 1.09 \\
\hline
\end{tabular}

We also assume in this case that the neutron star is non-spinning, but with $q=m_{\mathrm{NS}} / m_{\mathrm{BH}} \neq 0$. The curves of $R$ versus $f_{\mathrm{td}}$ are calculated using the formula given in equation. (3) of [36], for the same parameters as in that work: $m_{\mathrm{NS}}=1.4 M_{\odot}, m_{\mathrm{BH}}=2.5,10,20,40,80 M_{\odot}$. The circles over each curve specify where the plunge occurs for different values of $\chi_{\mathrm{BH}}$. Filled circles correspond to the extreme mass ratio approximation. The meaningful part of each curve will be that to the left of the white circles $\left(f_{\mathrm{td}}<f_{\text {plunge }}\right)$.

The plunge frequencies calculated in [36] for the case with $m_{\mathrm{BH}}=2.5 M_{\odot}$ (black circles in the right-hand-side curve of figure 5) correspond to retrograde orbits. However, for that mass ratio $(q=0.56)$, the test particle orbiting the merger product ISCO does so in a prograde fashion due to the orbital angular momentum dominating the final spin. Thus, the final spin is aligned with the orbital angular momentum even though its initial spin is anti-aligned.

We now compare with the NR results obtained in [40]. We calculate $f_{\mathrm{BKL}}$ for the six cases considered in that work, models A-F. The BKL frequencies are shown in table 1, together with the corresponding parameters $q$ and $M \equiv M_{\mathrm{BH}}+M_{\mathrm{NS}}\left(\chi_{\mathrm{BH}}\right.$ and $\chi_{\mathrm{NS}}$ are set to zero as in [40]). Although the models studied in [40] expand only a small range of parameters; we see that, at least in that range, the frequencies obtained with the BKL approach are consistent with the NR results (see figure 7 of [40]) — that is they predict in every case a higher value than the measured $f_{\text {cut }}$ caused by the tidal effects.

\section{Conclusion}

The estimate for the spin of the black hole that results from $\mathrm{BH}-\mathrm{BH}, \mathrm{BH}-\mathrm{NS}$, and some NS-NS mergers found in [13] leads to an estimate for the ISCO, light ring and quasinormal mode ringdown frequencies of a general compact binary system that is waveform model independent. Both frequencies have impact on searches for the inspiral, merger and ring-down epochs of the gravitational waves emitted by these systems. We have shown that the most interesting merger-epoch time-frequency volumes are for extreme mass ratios. More work may have to be done to model merger epochs for these systems. The formalism presented here to describe inspiral, merger and ring-down epochs can be extended to arbitrary spin configurations and may help guide the construction of better analytic models.

As for inspiral epoch searches alone, we have shown that for symmetric mass, nonspinning systems with greater than $45 M_{\odot}$ total mass there can be a $30 \%$ gain in SNR over using the Schwarzschild ISCO radius as is done currently [14] even including conservative phase errors. It is worth noting that more general fittings can be computed in order to cover the full range of parameters $\left(q, \chi_{1}, \chi_{2}\right)$ within the BKL approximation. Additionally, we discussed cases where the neutron star tidal disruption may be observed more easily through 
gravitational waves. We would like to stress that this work is open ended. Here we have only considered spins that are aligned and anti-aligned with the orbital angular momentum. It is certainly possible to extend this work to make predictions about more general cases, all of which should be compared with numerical relativity results as they are produced.

\section{Acknowledgments}

The authors would like to acknowledge L Lehner for suggesting this project and for invaluable discussions. The authors also thank L Lehner and F Pretorius for providing the test particle time of flight used in section 2. Gabriela González, Patrick Brady, Alessandra Buonanno and Jolien Creighton provided motivating discussions and insightful comments. C Hanna would like to thank the LIGO Scientific Collaboration Compact Binary Coalescence (LIGO CBC) working group. This work was supported in part by NSF grants PHY-0605496, PHY-0653369 and PHY-0653375 to the Louisiana State University and PHY-0603762 to the University of Maryland. $\mathrm{CH}$ would like to thank the Kavli Institute for Theoretical Physics, for their hospitality, where some of this work was completed. The Kavli Institute is supported by NSF grant PHY05-51164.

\section{References}

[1] Cutler C et al 1993 The last three minutes: issues in gravitational-wave measurements of coalescing compact binaries Phys. Rev. Lett. 702984

[2] Owen B J 1996 Search templates for gravitational waves from inspiraling binaries: choice of template spacing Phys. Rev. D 536749

[3] The LIGO Scientific Collaboration 2004 Analysis of LIGO data for gravitational waves from binary neutron stars Phys. Rev. D 69122001

[4] The LIGO Scientific Collaboration 2005 Search for gravitational waves from galactic and extra-galactic binary neutron stars Phys. Rev. D 72082001

[5] The LIGO Scientific Collaboration 2006 Search for gravitational waves from binary black hole inspirals in LIGO data Phys. Rev. D 73062001

[6] The LIGO Scientific Collaboration 2008 Search for gravitational waves from binary inspirals in S3 and S4 LIGO data Phys. Rev. D 77062002

[7] Buonanno A, Cook G B and Pretorius F 2007 Inspiral, merger and ring-down of equal-mass black-hole binaries Phys. Rev. D 75124018

[8] Berti E, Cardoso V, Gonzalez J A, Sperhake U, Hannam M, Husa S and Bruegmann B 2007 Inspiral, merger and ring-down of unequal mass black hole binaries: a multipolar analysis Phys. Rev. D 76064034

[9] Vaishnav B, Hinder I, Herrmann F and Shoemaker D 2007 Matched filtering of numerical relativity templates of spinning binary black holes Phys. Rev. D 76084020

[10] Baker J G, McWilliams S T, van Meter J R, Centrella J, Choi D, Kelly B J and Koppitz M 2007 Binary black hole late inspiral: simulations for gravitational wave observations Phys. Rev. D 75124024

[11] Pan Y et al 2008 A data-analysis driven comparison of analytic and numerical coalescing binary waveforms: non-spinning case Phys. Rev. D 77024014

[12] Ajith P 2008 Gravitational-wave data analysis using binary black-hole waveforms Class. Quantum Grav. 25 114033

[13] Buonanno A, Kidder L E and Lehner L 2008 Estimating the final spin of a binary black hole coalescence Phys. Rev. D 77026004

[14] Allen B, Anderson W G, Brady P R, Brown D A and Creighton J D E 2005 FINDCHIRP: an algorithm for detection of gravitational waves from inspiraling compact binaries arXiv:gr-qc/0509116

[15] Buonanno A and Damour T 1999 Effective one-body approach to general relativistic two-body dynamics Phys. Rev. D 59084006

[16] Buonanno A, Pan Y, Baker J G, Centrella J, Kelly B J, McWilliams S T and van Meter J R 2007 Toward faithful templates for non-spinning binary black holes using the effective-one-body approach arXiv:0706.3732

[17] Kidder L E, Will C M and Wiseman A G 1992 Innermost stable orbits for coalescing binary systems of compact objects Class. Quantum Grav. 9 L125-31 
[18] Blanchet L 2002 Innermost circular orbit of binary black holes at the third post-Newtonian approximation Phys. Rev. D 65

[19] Blanchet L 2006 Gravitational radiation from post-Newtonian sources and inspiralling compact binaries Living Rev. Rel. 94 (http://www.livingreviews.org/lrr-2006-4)

[20] Bardeen J M, Press W H and Teukolsky S A 1972 Rotating black holes: locally nonrotating frames, energy extraction, and scalar synchrotron radiation Astrophys. J. 178347

[21] Rezzolla L, Barausse E, Dorband E N, Pollney D, Reisswig C, Seiler J and Husa S 2008 On the final spin from the coalescence of two black holes Phys. Rev. D 78044002

[22] Rezzolla L, Diener P, Dorband E N, Pollney D, Reisswig C, Schnetter E and Seiler J 2008 The final spin from the coalescence of aligned-spin black-hole binaries Astrophys. J. 674 L29

[23] Sperhake U, Berti E, Cardoso V, Gonzalez J A, Bruegmann B and Ansorg M 2008 Eccentric binary black-hole mergers: The transition from inspiral to plunge in general relativity Phys. Rev. D 78064069

[24] Lehner L 2007 private communication

[25] Echeverria F 1989 Gravitational-wave measurements of the mass and angular momentum of a black hole Phys. Rev. D 40 3194-203

[26] Creighton J D E 1999 Search techniques for gravitational waves from black-hole ringdowns Phys. Rev. D 60022001

[27] Berti E, Cardoso V and Will C M 2006 On gravitational-wave spectroscopy of massive black holes with the space interferometer LISA Phys. Rev. D 73064030

[28] Abbott B et al 2007 Search for gravitational-wave bursts in LIGO data from the fourth science run Class. Quantum Grav. 24 5343-69

[29] Anderson W G, Brady P R, Creighton J D E and Flanagan E E 2001 An excess power statistic for detection of burst sources of gravitational radiation Phys. Rev. D 63042003

[30] Baumgarte T, Brady P, Creighton J D E, Lehner L, Pretorius F and DeVoe R 2008 Learning about compact binary merger: the interplay between numerical relativity and gravitational-wave astronomy Phys. Rev. D 77084009

[31] Finn L S and Chernoff D 1993 Observing binary inspiral in gravitational radiation: One interferometer Phys. Rev. D 47 2198-219

[32] Flanagan É É and Hughes S A 1998 Measuring gravitational waves from binary black hole coalescences: I. Signal to noise for inspiral, merger, and ring-down Phys. Rev. D 57 4535-65

[33] Boyle L, Kesden M and Nissanke S 2008 Binary black hole merger: symmetry and the spin expansion Phys. Rev. Lett. 100151101

[34] Damour T, Iyer B R and Sathyaprakash B S 2001 Comparison of search templates for gravitational waves from binary inspiral Phys. Rev. D 63044023

[35] LIGO Science Requirement Document noise curve http://www.ligo.caltech.edu/jzweizig/distribution/ LSC_Data/srd.html

[36] Vallisneri M 2000 Prospects for gravitational-wave observation of neutron-star tidal disruption in neutronstar/black-hole binaries Phys. Rev. Lett. 843519

[37] Saijo M and Nakamura T 2001 Possible direct method to determine the radius of a star from the spectrum of gravitational wave signals II : spectra for various cases. Phys. Rev. D 63064004

[38] Lee L 1992 Determining the nuclear equation of state from neutron-star masses and radii Astrophys. J. 398 569-73

[39] Harada T 2001 Reconstructing the equation of state for cold nuclear matter from the relationship of any two properties of neutron stars Phys. Rev. C 64048801

[40] Shibata M and Taniguchi K 2008 Merger of black hole and neutron star in general relativity: Tidal disruption, torus mass, and gravitational waves Phys. Rev. D 77084015

[41] Hannam M, Husa S, Gonzalez J A, Sperhake U and Bruegmann B 2008 Where post-newtonian and numericalrelativity waveforms meet Phys. Rev. D 77044020

[42] Baker J G, van Meter J R, McWilliams S T, Centrella J and Kelly B J 2007 Consistency of post-Newtonian waveforms with numerical relativity Phys. Rev. Lett. 99181101

[43] Boyle M et al 2007 High-accuracy comparison of numerical relativity simulations with post-Newtonian expansions Phys. Rev. D 76124038

[44] Gopakumar A, Hannam M, Husa S and Brügmann B 2007 Comparison between numerical relativity and a new class of post-Newtonian gravitational-wave phase evolutions: the non-spinning equal-mass case arXiv:0712.3737

[45] Hannam M, Husa S, Brügmann B and Gopakumar A 2007 Comparison between numerical-relativity and post-Newtonian waveforms from spinning binaries: the orbital hang-up case arXiv:0712.3787 